\title{
Dermatology and Dermatologic
}

\section{Rare Case Report of Angiolymphoid Hyperplasia with Eosinophilia}

\section{RK Belkharoeva, AR Hubail ${ }^{*}$, NP Tepljuk, OY Olisova, VB Pinegin and VA Koroleva}

I.M. Sechenov First Moscow State Medical University, Russian Federation

*Corresponding author: AR Hubail, Dermatology Resident, I.M. Sechenov First Moscow State Medical University, Russian Federation, Tel: +7 985759 85 34 ; E-mail: dr.amalhubail@gmail.com

Received date: November 27, 2017; Accepted date: January 15, 2018; Published date: January 22, 2018

Copyright: (c) 2018 Belkharoeva RK, et al. This is an open-access article distributed under the terms of the Creative Commons Attribution License, which permits unrestricted use, distribution, and reproduction in any medium, provided the original author and source are credited.

\begin{abstract}
Aims and objective: The aim of this study was to assess the clinical and demographic features of a rare cutaneous condition, angiolymphoid hyperplasia with eosinophilia (ALHE), and compare them with those previously reported. ALHE is a benign vascular proliferation of unknown etiology and its treatment is unclear.

Methods: A 29 year old woman visited the Hospital of Skin and Venereal diseases for pruritic lesion in the ear. She presented with a reddish papulonodular rash. Laboratory tests showed no abnormalities. She was diagnosed with ALHE based on clinical, histological, and immunological features. Treatment with injectable prolonged systemic glucocorticoid and topical prednisolone was initiated. The rash and pruritic sensation improved within one week in the hospital, and the reddish infiltration and nodules had dramatically decreased at the 6 month follow-up. Continuous topical application resulted in no recurrence at the 6 month follow-up.

Results and conclusion: Management of ALHE is still poorly standardized and poorly understood due to uncertainties concerning its pathophysiology and development and the small number of available studies. Establishing the final clinical diagnosis is difficult owing to the absence of subjective sensations. ALHE can persist for years but serious complications like cancer transformation do not occur.
\end{abstract}

\section{Keywords: Kimura's disease; Angiolymphoid hyperplasia; Case Report}

Eosinophilia; Cutaneous neoplasms

\section{Introduction}

Angiolymphoid hyperplasia with eosinophilia (ALHE) is a vasoproliferative idiopathic condition that represents a reactive hyperplasia of blood vessels with signs of arteriovenous anastomoses [1-3]. It manifests as nodular plaques or papules mostly around the head and neck, and rarely in other areas, such as the breasts, penis, hands, scrotum, shoulders and oral mucosa. Kimm and Szeto first discovered this disease in 1937 [1], reporting 7 cases of hyperplastic eosonophilic granuloma. In 1948, Kimura et al. [3] described it as an unusual granulomatosis combined with hyperplastic changes in the lymphoid tissue and eosinophilia. It has since been called Kimura's disease (KD) [4-11]. However, in 1969, Wells and Whimster [12] presented a case with clinical and morphological characteristics similar to those of $\mathrm{KD}$, calling it subcutaneous ALHE. To date, few studies have reported that KD and ALHE are different clinical variants of the same disease, indicating that ALHE is an arteriovenous disease with secondary inflammation and $\mathrm{KD}$ is an inflammatory process with secondary vascular proliferation $[4,5]$.

Based on the literature, ALHE occurs in women and middle-aged Asian men, and is more common in young women with positive allergic anamnesis (bronchial asthma, pollinosis and atopic dermatitis) $[6,7]$. However, its etiology remains unknown.

\section{Presenting history}

A 29 year old woman visited the Rakhmanova Skin and Venereal Diseases Hospital of Sechenov First Moscow State Medical University because of pruritic rashes on the tragus of the outer ear and behind the ear present for many years. The rashes were unaccompanied by subjective sensations.

Based on her past medical history, the first rash appeared 5 years prior her visit. The patient did not self-treat or seek medical advice. Since then, the lesions grew slowly, without disturbing the patient or causing any discomfort. Clinical and biochemical blood tests and general urine analysis did not show any irregularities. Test results for a complex of hemotransmissive infections were negative. Computed tomography scan of the chest and abdominal ultrasound revealed the absence of any pathology.

\section{Clinical examination}

On physical examination, multiple papular lesions localized in the right tragus of the outer ear were noted. These monomorphic papules were $\sim 5 \mathrm{~mm}$ in diameter, hemispherical in shape, not sharply demarcated from the surrounding healthy skin, reddish-blue in color and of solid consistency. The skin integuments outside the lesions were pale pink, clean, and moderately humid. Turgor and elasticity were in accordance with age. The mucous membranes, hair, and nail plates of the hands and feet were not affected. Marked enlargement of the lymph node of up to $2.5 \mathrm{~cm}$ was noted posterior to the auricle on the same side; the skin over the lymph node was unchanged. The 
remaining lymph nodes were not palpable. No other subjective findings were noted (Figure 1).
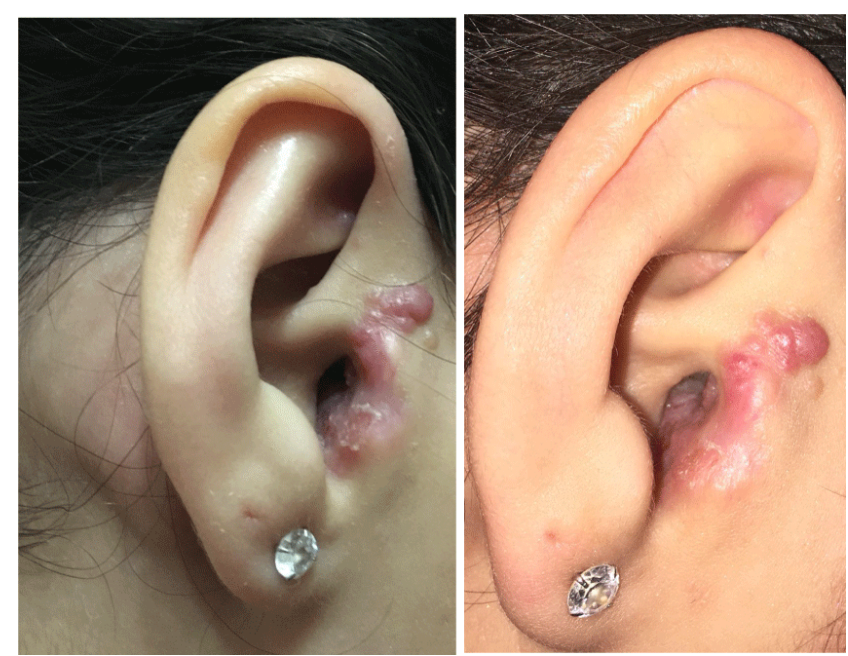

Figure 1: Multiple papular lesions on the patient's ear on presentation.

\section{Histopathological investigations}

A skin biopsy specimen was obtained, and histopathological findings were of parakeratosis, acanthosis, spongiosis with the formation of intradermal vesicles with neutrophilic granulocytes and vacuolar degeneration of keratinocytes. The dermis had signs of edema and moderately marked lymphoid proliferation of small blood vessels, which were small, with round, oval, and irregular nuclei; moreover both histiocytes and eosinophilic granulocytes were identified perivascularly and periglandularly. Perivascular infiltrate consisted mainly of lymphocytes without obvious signs of epidermotropism and eosinophils (Figure 2).

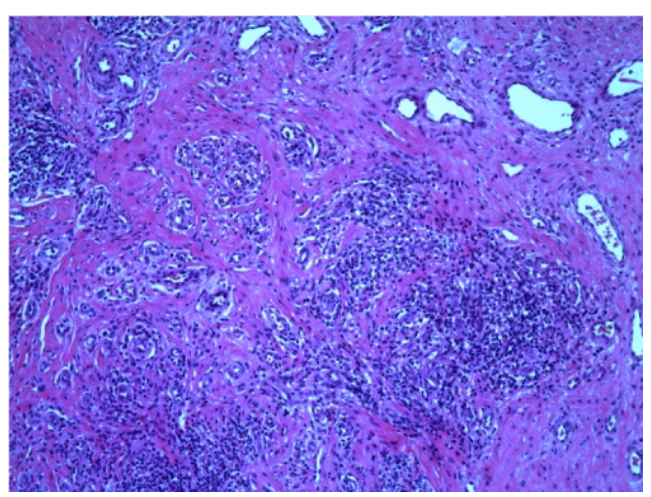

Figure 2: Histology slide from patient's skin biopsy showing proliferation of small blood vessels in the dermis, perivascular infiltrate consisting mainly of lymphocytes without obvious signs of epidermotropism and eosinophils.

\section{Immunohistochemical investigations}

Immunohistochemical study revealed mostly positive CD34 and CD31 reactions of the endothelium in numerous vessels and only a few were positive for alpha CD79, indicating the predominance of $\mathrm{T}$ lymphocyte infiltration. The proliferative activity of Ki-67 in the endothelial cells was low. E-cadherin in the spongy areas of altered epithelial layer was immunoreactive. Thus, no elements of tumor growth of stroma fibrosarcoma were detected (Figure 3).

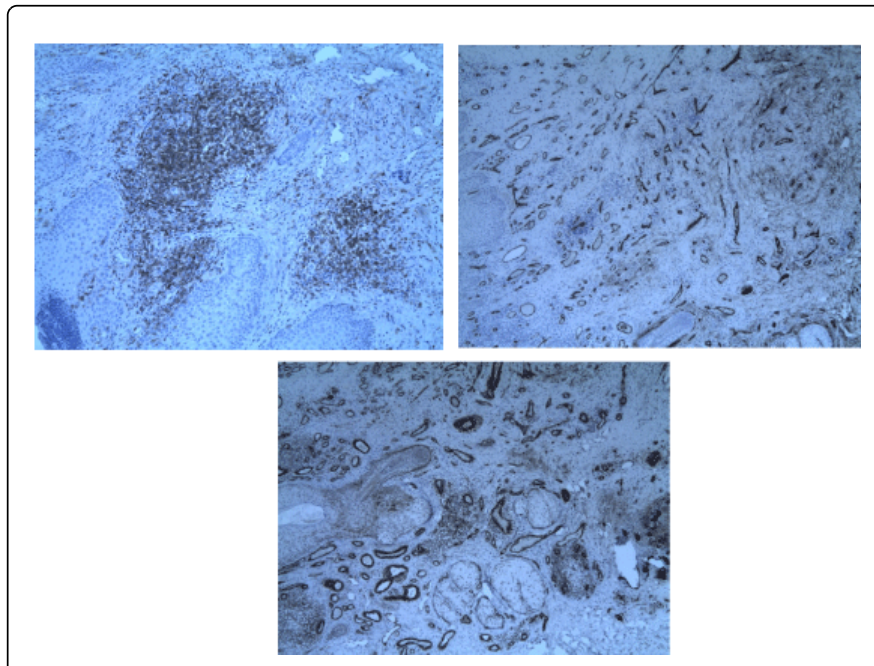

Figure 3: Immunohistochemistry study of $\mathrm{CD} 4+, \mathrm{CD} 3+$ and actin respectively, showing positive results, indicating predominance of T-lymphocyte infiltration.

\section{Treatment}

Based on the medical history, physical examination, and pathohistological data, we established a diagnosis of ALHE. Treatment was initiated using prolonged systemic glucocorticoid, betamethasone sodium phosphate and betamethasone acetate injectable suspension 30 $\mathrm{mg} / 5 \mathrm{~mL}(6 \mathrm{mg} / \mathrm{mL})$ administered at $1 \mathrm{ml}$ per week for 2 weeks. The concomitant therapy included $0.5 \%$ topical prednisolone.

The size of the infiltrate was reduced by $60 \%$ after in-hospital admission and treatment for 1 week. The patient was discharged with recommendations to continue treatment with prolonged topical therapy. Informed consent was obtained on admission.

\section{Follow up}

At the 3 month follow-up, there was concern that the lesions might have relapsed or remained static without improvement, but the lesions significantly regressed. At the 6 month follow-up, the lesions regressed by approximately $70 \%$ in comparison to the time of presentation (Figure 4). 


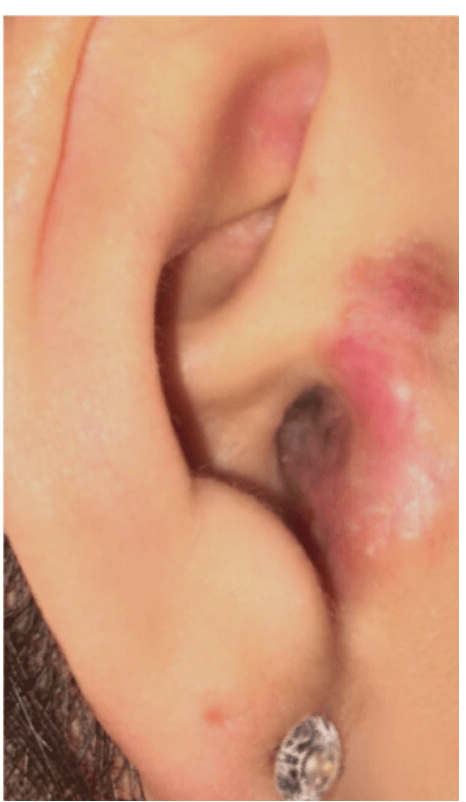

Figure 4: Lesions at 6 month follow-up showing great improvement.

\section{Discussion}

ALHE is generally considered a benign reactive lymphoproliferative process, causing the formation of a localized non-proliferating lesion with small blood vessels surrounded by white blood cells (lymphocytes and eosinophils). The cause of ALHE is unknown. Existing theories link it to injury or insect bites [5].

The diagnosis of ALHE is based on biopsy and clinical presentation. Clinically, it is characterized by small brownish papules, $2-5 \mathrm{~mm}$ in diameter, clustered on a background of normal skin surface, with or without slight peeling. The rash is frequently localized on the neck and scalp; exposure of regional lymph nodes and salivary glands is also possible. The lesions may be pruritic, asymptomatic, or painful. Uncommon symptoms include bleeding and pulsation [8].

Histology results reveal hyperplasia of small blood vessels lined with rounded endothelial cells, with abundant cytoplasm and sometimes atypical nucleus; the vessels are similar to the endothelial venules of lymphoid tissue. The dermis is inflamed with increased vascularity [6]. Direct immunofluorescence shows fixation of IgA and IgM and the C3 component of the complement in the walls of the skin blood vessels. Complete blood count may show eosinophilia in up to $20 \%$ of cases only. Urinalysis may be also considered because renal disease occurs in some patients with ALHE rarely $[5,9]$.

The differential diagnosis includes pseudolymphomas (lymphoplasia), sinus histiocytosis with massive lymphadenopathy, pyogenic granulomas, hemangiomas, soft tissue tumors, angiosarcoma, Kaposi sarcoma, bacillary angiomatosis, bartonellosis, sarcoidosis, metastatic carcinoma, cutaneous B-cell lymphoma, and other lymphomas. No unified approach exists for ALHE (KD) treatment.

The treatment depends on the size of the lesion and chronicity. If the lesion is small, observation for 3-6 months is recommended due to a chance of spontaneous regression. If the lesion is large, then surgical excision of the tumor tissues is suggested; however, relapses were observed in most patients who underwent surgery, particularly with incomplete excision. Mohs micrographic surgery with excision of the abnormal vessels has been proven to be very effective. If surgery is contraindicated, treatment of relapses and resistant disease is performed by glucocorticosteroid and local radiation therapies (25-30 Gy). One study reported that the clinical effect of radiotherapy was achieved in $74 \%$ of their patients. The most commonly used radiation therapy is pulsed dye laser. Other modes of ALHE treatment include systemic, intrafocal and topical corticosteroids, electro-dessication, cryotherapy and hormonal therapies. ALHE recurrence was reported in the literature after discontinuation of hormonal therapy, such as imiquimod ointment [10-15].

Research has shown good insight with treatment using oral propranolol, with no recurrence reported after its use. Using topical timolol is another modality that has been tried with some success. Despite the multiplicity of therapeutic modalities, their real efficacy has not been well studied, and frequent recurrences have been noted [10-15].

Prognosis is variable. Even with surgery, approximately $41 \%$ failure rate is observed. This condition generally persists for years, but complications actually rarely occur and these may include nephropathy [10].

\section{Conclusion}

We present a rare cutaneous dermatosis, wherein establishing the final clinical diagnosis of this disease was difficult owing to the absence of subjective sensations and overall undisturbed condition despite the presence of the disease for several years. In the future, patients with this diagnosis must be constantly followed up by a dermatologist to prevent the transformation process. This article lays the grounds for future research, particularly on early detection of the condition as it is misdiagnosed at first in most cases.

\section{References}

1. Kim HT, Szeto C (1937) Eosinophilic hyperplastic lymphogranuloma, comparison with Mikulicz's disease [in Chinese]. Chin Med J 23: 699-700.

2. Armstrong WB, Allison G, Pena F, Kim JK (1998) Kimura's disease: two case reports and a literature review. Ann. Otol Rhinol Laryngol 107: 1066-1071.

3. Kimura's T, Yoshimura S, Ishikawa E (1948) On the unusual granulation combined with hyperplastic changes of lymphatic tissues. Trans Soc Pathol Jpn 37: 179-180.

4. Carlesimo M, Mari E, Tammaro A, Persechino S, Camplone G. (2007) Angiolymphoid hyperplasia with eosinophilia treated with isotretinoin. Eur J Dermatol 17: 554-555.

5. Larroche C, Blétry O (2005) Kimura’s disease. Orphanet Encyclopedia.

6. Wolff K, Goldsmith LA, Katz SI, Gilchrest B, Am Paller AS et al. (2012) Angiolymphoid hyperplasia with eosinophilia and Kimura disease. Fitzpatrick Dermatology in General Medicine, McGraw Hill Medical, USA.

7. Olisova O, Potekaev N (2013) Cutaneous pseudolymphoma. Practice 7: 1-138.

8. Isohisa T, Masuda K, Nakai N, Takenaka H, Katoh N (2014) Angiolymphoid hyperplasia with eosinophilia treated successfully with imiquimod. Int J Dermatol 53: e43-e44.

9. Adler BL, Krausz AE, Minuti A, Silverberg JI, Lev-Tov H (2016) Epidemiology and treatment of angiolymphoid hyperplasia with eosinophilia (ALHE): A systematic review. J Am Acad Dermatol 74:506-512. 
Citation: Belkharoeva RK, Hubail AR, Tepljuk NP, Olisova OY, Pinegin VB, et al. (2018) Rare Case Report of Angiolymphoid Hyperplasia with Eosinophilia. J Dermatol Dis 5: 269. doi:10.4172/2376-0427.1000269

Page 4 of 4

10. Wanat K, James A (2010) Kimura disease: Treatment and medication. Emedicine, Accessed on 21 August 2017.

11. Kohli A, Singh G (2008) Kimura's disease. JK Sci 10: 149-150.

12. Wells GC, Whimster IW (1969) Subcutaneous angiolymphoid hyperplasia with eosinophilia. Br J Dermatol 81:1-14.

13. Ivanov OL (2007) Skin and venereal disease: A Handbook. In: Medicine, Moscow, pp: 118-119.
14. Horst C, Kapur N (2014) Propranolol: A novel treatment for angiolymphoid hyperplasia with eosinophilia. Clin Exp Dermatol 39: 810-812.

15. Chacon AH, Mercer J (2016) Successful management of angiolymphoid hyperplasia with eosinophilia in a split-face trial of topical tacrolimus and timolol solution. G Ital Dermatol Venereol 15: 436-440. 